Active controls of independently-rotating wheels with gyro and tachometers - Simple solutions for perfect curving and high stability performances

Xiaoyuan Liu*, Roger Goodall and Simon Iwnicki

Institute of Railway Research, University of Huddersfield, Huddersfield, UK

*corresponding author: x.liu@hud.ac.uk 


\title{
Active control of independently-rotating wheels with gyro and tachometers - Simple solutions for perfect curving and high stability performances
}

\begin{abstract}
Although a passive independently-rotating wheel-pair (IRW) is not well suited for curve negotiation due to an inability to provide the necessary steering, the active IRW system offers the potential to realize perfect curving with a satisfactory running stability. Simple control solutions to realize automatic steering and stability of the IRW system are proposed in this paper. In this control approach, only gyroscopes and tachometers are adopted for the measurements. Associated with a prototype of the two-axle IRW vehicle, the active control strategies for both motor-driven and actuator-driven solutions are illustrated. The simulation results demonstrate significant alleviations in the wheel/rail interactions with perfectcurving performances and superior running stabilities owing to the proposed control strategies.
\end{abstract}

Keywords: independently-rotating wheels; active control; curve negotiation; stability; dual-loop control

\section{Introduction}

Compared with the solid-axle wheelset of a railway vehicle, the passive independentlyrotating wheel (IRW) has almost no capacity to provide the steering needed for curve negotiation. This feature can result in severe wheel/rail wears and very high wheel/rail contact forces due to the occurrence of flange contact in curves, even at low speeds. These factors hinder many further applications of the IRW system, even though IRW systems have many distinct advantages including simplified stability control [1], lower centre-ofgravity of the vehicle and low-cost maintenance. To improve the dynamic performance of the IRW vehicle, relevant active controls can be applied by the use of independentlydriven motors or linear actuators [2-5], both of which are considered and compared within this paper.

\subsection{Motor control}

The principle of the motor control approach is to apply opposite rotational torques of the same value (differential torques) to the wheels of the same axle, which may be 
superimposed on a common traction and braking torque. The differential torques lead to differences in the rotational speeds of the wheels. The differential velocity of the wheels generates the yaw torque needed for steering by the longitudinal creep, which is similar to a solid axle wheelset except that generally a much lower primary yaw stiffness can be used. It is feasible to control the differential velocity from the motor torque input by a fast closed control loop, because a high bandwidth control can be realized by modern motors. The output, differential velocity of two wheels, can be obtained by two tachometers measuring the rotational velocities of the wheels. The differential loop, the closed-loop to control the differential velocity of wheel-pair, with a zero input in some senses replicates a solid-axle connection and hence provides the steering capacity of the independently-driven IRW system.

However, the differential loop itself is not a stable system due to the wheel tread conicity. There are various possibilities for the control signals for providing the control commands for the wheel differential velocities. Several researchers have discussed these signals, including wheel-pair attack angle [6], wheel/rail lateral displacement [7], etc. Certain output quantities are able to contribute to the guidance of the IRW, however, unstable motions resulting from the differential control can still lead to oscillations of the wheel-pair. Besides, the difficulty of the output measurement is another important issue involving the availability and the cost of the sensor technology.

\subsection{Linear actuator control}

Unlike the solid-axle wheelset or motorized IRW, for the non-motorized IRW it is not possible to steer the non-motorized IRWs via the longitudinal creep forces. In contrast, the non-motorized IRW requires an external controlled force/torque to guide the wheelpair. The concept of actuator control is to apply the required steering torque directly to the axle-bridge of the wheel-pair in the yaw direction via a pair of linear actuators, which are connected with the carbody or bogie frame. The controlling of the wheel-pair yaw motion can be a very efficient way to steer and stabilize the IRW system. Since the two wheels can move relatively from each other, the actuation force needed can be smaller than for the solid-axle wheelset. Furthermore, the pure rolling of the wheels can be readily realized with this approach. In this configuration, the longitudinal creep torque does not exist in the wheel/rail interface any longer. This feature contributes to the elimination of the hunting motion of the vehicle, which is a significant advantage of this control 
approach.

Under this circumstance, a stable steering control is the focus of this research, since it is relatively simple to solve the kinetic instability of the IRW by introducing active or passive damping. In [8,9], a general steering and stability control approach for the actuator-steered IRW system is proposed using a differential velocity control and an actuator velocity control, however, an effective control strategy to eliminate the interference between the steering and stability controls still needs to be investigated. In [10], the steering control method for keeping the wheel-pair at the track centreline is discussed. Similar to the motor control approach, it is also necessary to avoid difficult measurements and complicated control methods for this actuator control approach.

\section{Novel Control Solutions}

A general lateral model of the independently-rotating wheel-pair is comprehensively discussed in [1]. Since the design of the control system is highly dependent upon the parameter values of the physical system, the numerical experiments are very useful in providing straightforward solutions for the control design. From a series of numerical analyses, it is found that the yaw angular velocity of the axle-bridge and the differential velocity of the wheel pair are highly correlated, but their controls can be developed dependently if a proper control strategy is selected. Correspondingly, the control options for motor-steered and actuator-steered approaches are investigated in association with a prototype of a two-axle IRW vehicle.

\subsection{Motor control}

As mentioned above, the stabilization of the differential loop is the main issue for the motor control approach. Here a simple control scheme is proposed to solve the stability problem associated with a two-axle vehicle with a low yaw stiffness. In this scheme, the measurement of the yaw angular velocity of the axle-bridge by a mono-axial gyro is introduced to stabilize the differential loop. Figure 1 demonstrates the general layout of this dual-loop control scheme based on PID control. 


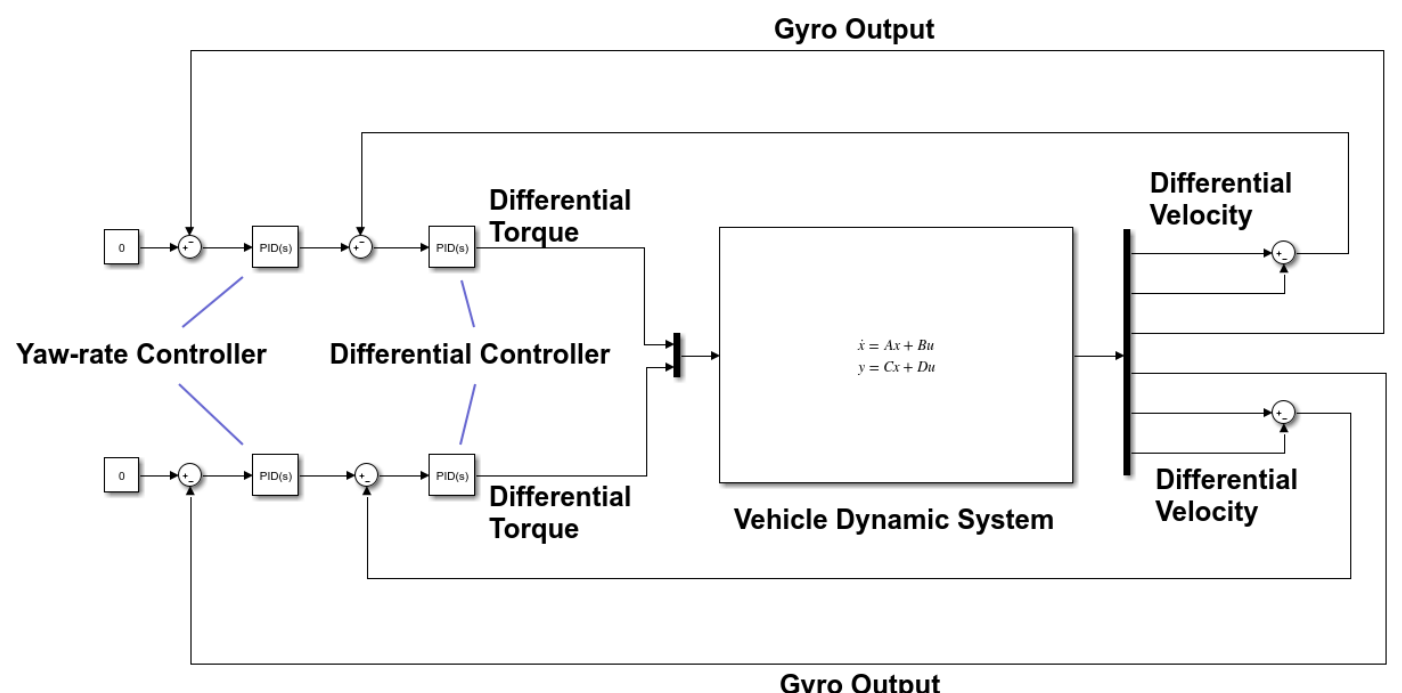

Figure 1. Dual-loop control scheme for a motor-steered two-axle IRW vehicle

In Figure 1, the yaw-rate control loop is based on the 'ground-hook damping' control concept. 'Ground-hook damping' means the unsprung mass is linked to the ground by an imaginary damper related to the absolute vibration velocity. The yaw-rate control functions as a stabilizer for the differential loop and keeps the wheel-pair in the right position of the track without undamped oscillations. Since the sensors of this control concept are achievable at relatively low costs, it is named as 'Practical dual-loop control' in this paper. Furthermore, another control loop with the wheel-pair lateral displacement selected as the feedback signal can be added to control the yaw-rate loop, forming a 'triloop control' system. With this approach, the wheel-pair is able to stay at the track centreline during curve negotiation. However, complicated techniques are needed at present to measure wheel-rail lateral displacement, such as advanced pattern recognition, a laser scanner, which may involve high costs and reliability problems.

\subsection{Actuator control}

In the motor driven approach, differential control is utilized for the steering of the wheelpair, while active damping in the yaw motion is employed in the stability control. In this sense, it is reasonable and feasible to consider applying a similar control principle to the actuator-steered IRW system by formulating a new practical dual-loop control. By identifying the properness of the transfer function (the degree of the numerator should not exceed the degree of the denominator), it is found that the yaw-rate control can function as the inner stability control loop with a fast response, while the differential control can work as the outer loop to steer the wheel-pair. In this configuration, the 
general dual-loop control scheme for the actuator-steered approach based on the PID control is shown in Figure 2.

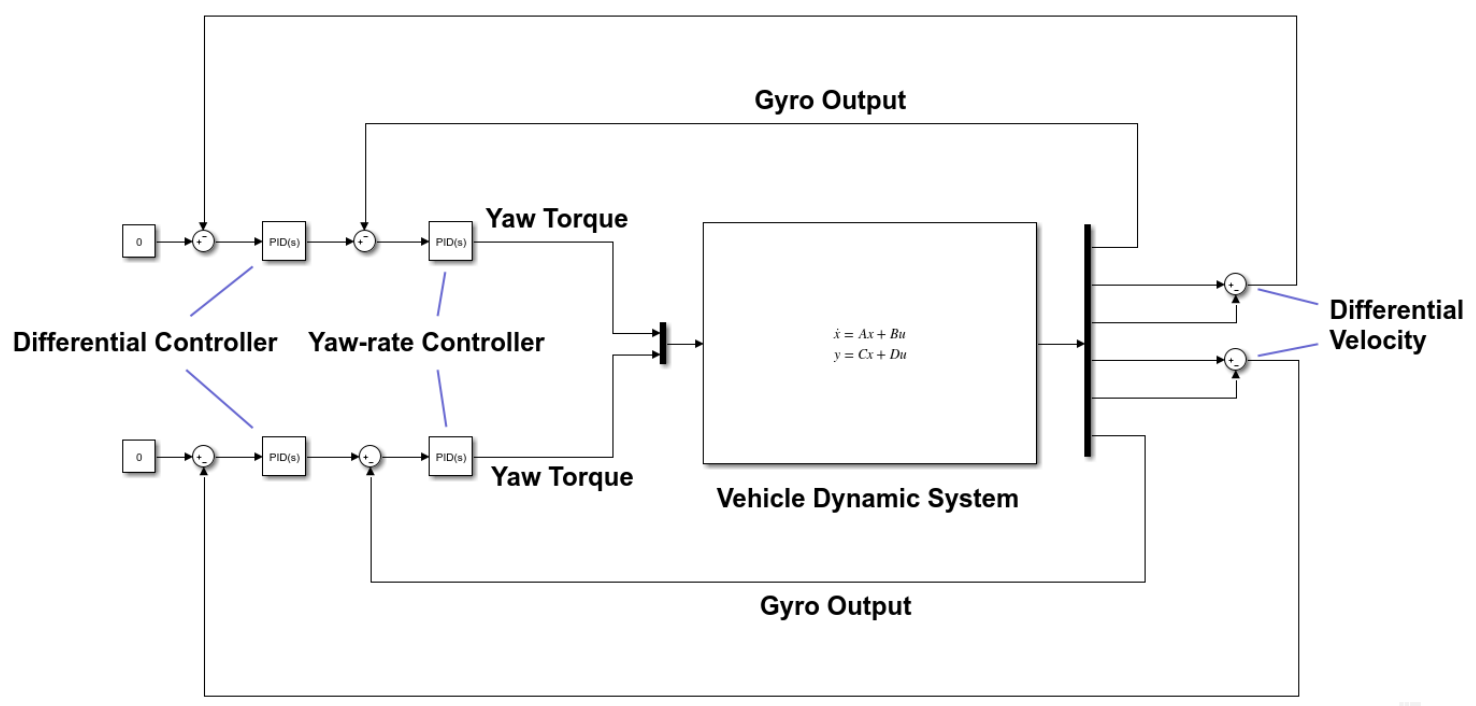

Figure 2. Dual-loop control scheme for an actuator-steered two-axle IRW vehicle

In this control strategy, the differential loop is utilized to keep the wheel-pair in the rolling position on the track with almost the same rotational velocity of the two wheels, mainly at low frequencies. Meanwhile, the yaw-rate control is applied to stabilize the unstable motion by the differential control and the natural kinematic instability of the IRW. During the curve negotiation, the controlled yaw torque steers the wheel-pair with a small attack angle generating a lateral creep force to counterbalance the uncompensated centrifugal force. In fact, the lateral creep force guides the IRW wheel-pair to the pure rolling position with a right rolling radius difference for the curve. In the whole process, longitudinal creep torque is no longer involved in the steady curve negotiation.

\subsection{Fundamental of controller tuning}

For the PID tuning, the transfer functions required can be obtained by transforming a simplified linear state-space model according to the input and outputs. As shown in Figure 3(a), for the motor driven approach, the PID tuning of the inner loop is on basis of the transfer function of differential velocity $V_{\mathrm{d}}(\mathrm{s})$ over differential torque $T_{\mathrm{d}}(\mathrm{s})$; the outer PID tuning is based on the transfer function of axle-bridge's yaw velocity $\dot{\psi}_{\mathrm{B}}(\mathrm{s})$ over differential velocity $V_{\mathrm{d}}(\mathrm{s})$ multiplied by the inner loop transfer function. Similarly in Figure 3(b), for the actuator-steered approach, the PID tuning of the inner loop is on basis of the transfer function of axle-bridge's yaw velocity $\dot{\psi}_{\mathrm{B}}(\mathrm{s})$ over yaw torque $T_{\mathrm{y}}(\mathrm{s})$; the 
outer PID tuning is based on the transfer function of differential velocity $V_{\mathrm{d}}(\mathrm{s})$ over axlebridge's yaw velocity $\dot{\psi}_{\mathrm{B}}(\mathrm{s})$ multiplied by the inner loop transfer function. In despite of different control configurations, the high-bandwidth controls should be implemented in the inner loops with larger PI values to realize fast responses of the actuation systems. Meanwhile, much lower bandwidth controls can be utilized in the outer loop with small PI values. The choice of the PID value should be determined by the final vehicle dynamics simulation result. If the control gain is too large, the control system can be unstable. On the contrary, if the PID gain is too small, the reaction of the control system can be slow and result in an inefficient dynamic performance.

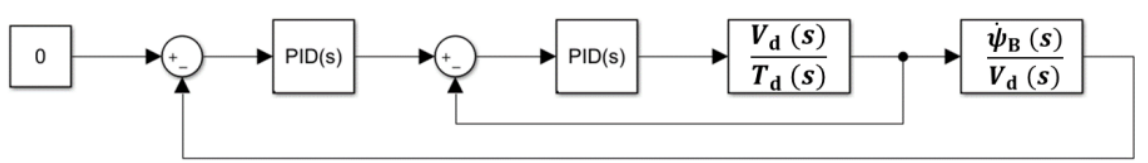

(a)

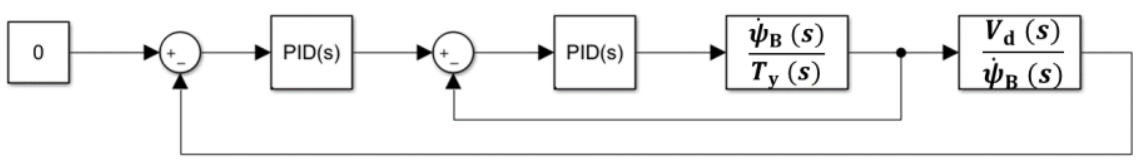

(b)

Figure 3. General diagrams for PID controller tunings. (a) Motor-steered approach; (b) Actuator-steered approach.

\section{Simulations and Analyses}

In order to test the feasibilities of the control strategies proposed, the Simpack-Simulink co-simulation is utilized here regarding a virtual prototype of two-axle IRW vehicle. In this two-axle vehicle with an axle load of $11 \mathrm{t}$, only a single stage of suspension is included in the suspension system. Several key dynamic parameters of the two-axle vehicle are listed in Table 1. Figure 4 demonstrates the general layouts of these IRW wheel-pair assemblies associated with actuation approaches.

Relatively low yaw stiffness is chosen for the vehicle in order to realize perfect curving by the introduction of the active steering systems. For the yaw stiffness, a higher value of $0.9 \mathrm{MNm} / \mathrm{rad}$ is adopted in the motor-steered vehicle, while a very low value of $0.15 \mathrm{MNm} / \mathrm{rad}$ is used in the actuator-steered vehicle. This is due to the influence of the 
actuation method on the vehicle stability, which will be explained in the following analyses. As the vehicle is equipped with the active steering technique, the wheel profile with a larger flange clearance can be applied due to the reduced flange wear on the curve. The British wheel profile BR-P8 with a flange clearance of $9.3 \mathrm{~mm}$ is adopted in this vehicle model.

Table 1. Main dynamic parameters of the two-axle IRW vehicle model.

\begin{tabular}{lll}
\hline Notation & Parameter & Value \\
\hline$I_{\mathrm{B} z z}$ & Yaw inertia of axle bridge & $200 \mathrm{kgm}^{2}$ \\
$I_{\mathrm{W} y y}$ & Rotational inertia of wheel & $50 \mathrm{kgm}^{2}$ \\
$R$ & Wheel radius & $0.43 \mathrm{~m}$ \\
$L$ & Wheel base & $8 \mathrm{~m}$ \\
$K_{\mathrm{pxM}}$ & Primary longitudinal stiffness for motor driven & $1.8 \mathrm{MN} / \mathrm{m}$ \\
& vehicle (per side) & \\
$K_{\mathrm{pxA}}$ & Primary longitudinal stiffness for actuator driven & $0.3 \mathrm{MN} / \mathrm{m}$ \\
& vehicle (per side) & \\
$K_{\mathrm{py}}$ & Primary lateral stiffness (per axle) & $0.3 \mathrm{MN} / \mathrm{m}$ \\
$d_{\mathrm{px}}$ & Lateral distance between longitudinal springs & $1 \mathrm{~m}$ \\
\hline
\end{tabular}

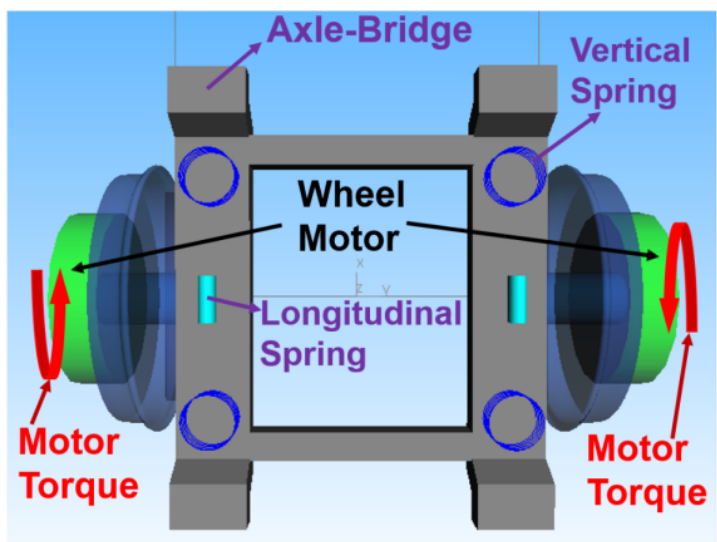

(a)

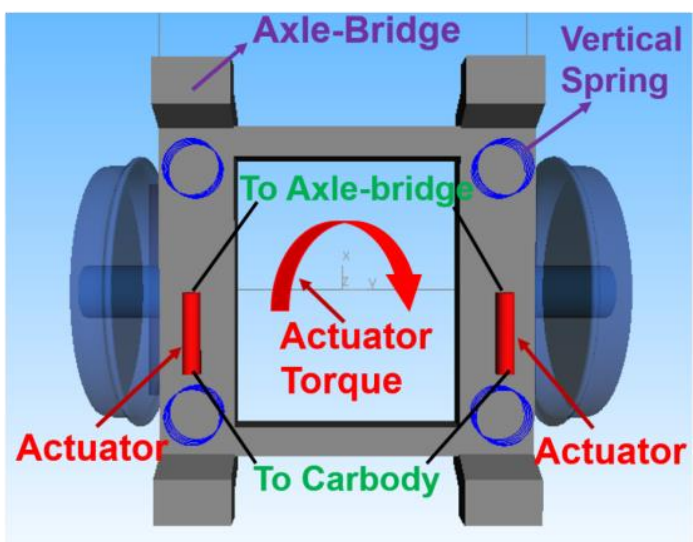

(b)

Figure 4. General layouts of IRW wheel-pair assemblies for different actuation approaches. (a) Motor-steered approach; (b) Actuator-steered approach. 
Regarding the vehicle dynamic parameters previously defined, Figure 5 exhibits the bandwidth tuning results of the outer control loops for both control strategies, which are derived from their corresponding linear models.

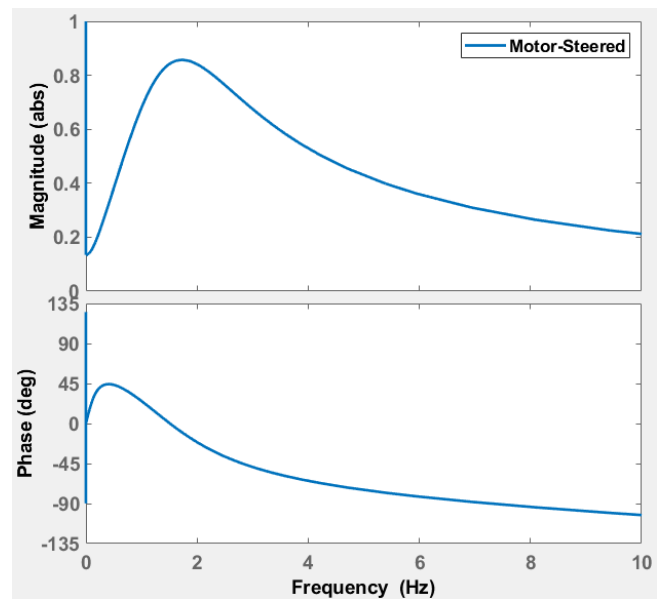

(a)

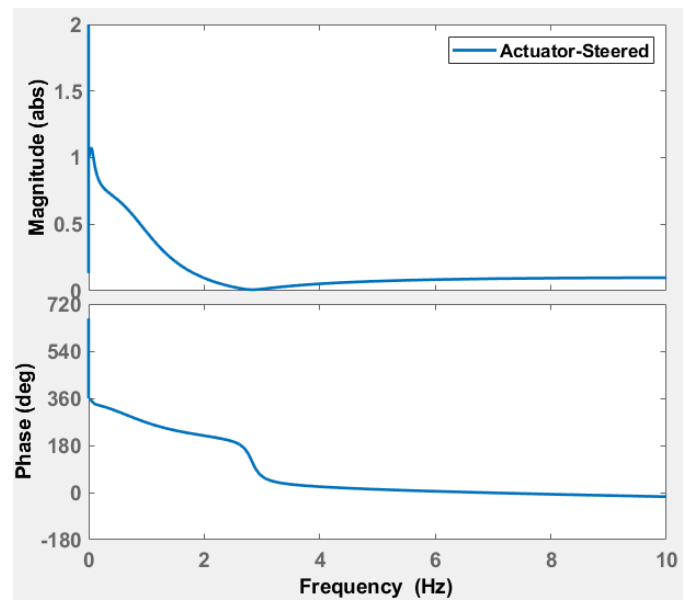

(b)

Figure 5. Bode diagrams of outer PID control loops for both actuation approaches. (a) Motor-steered approach; (b) Actuator-steered approach.

\subsection{Curve negotiation performance}

A few track cases are adopted to test the curving performance of the active control approaches for IRW vehicles. The track cases include a $250 \mathrm{~m}$ radius curve with a cant of $150 \mathrm{~mm}$ and a very short transition of $60 \mathrm{~m}$.

Figure 6 shows the dynamic behaviour of the two-axle IRW vehicle without the active steering during the negotiation of the $250 \mathrm{~m}$ curve at $72 \mathrm{~km} / \mathrm{h}$, corresponding to a cant deficiency condition with an uncompensated lateral acceleration of $0.65 \mathrm{~m} / \mathrm{s}^{2}$. The yaw stiffness utilized for this vehicle is $0.9 \mathrm{MNm} / \mathrm{rad}$, however, the simulation results for the yaw stiffness of $0.15 \mathrm{MNm} / \mathrm{rad}$ are very similar. It can be seen from Figures 6(a)-(d) that for a passive vehicle the lack of steering results in severe wheel/rail interactions with very large track forces and serious wheel/rail wears, including flange wears. The attack angles are very large due to the lack of the steering function for the passive IRW with an understeering of the leading wheel-pair and an oversteering of the trailing one. 


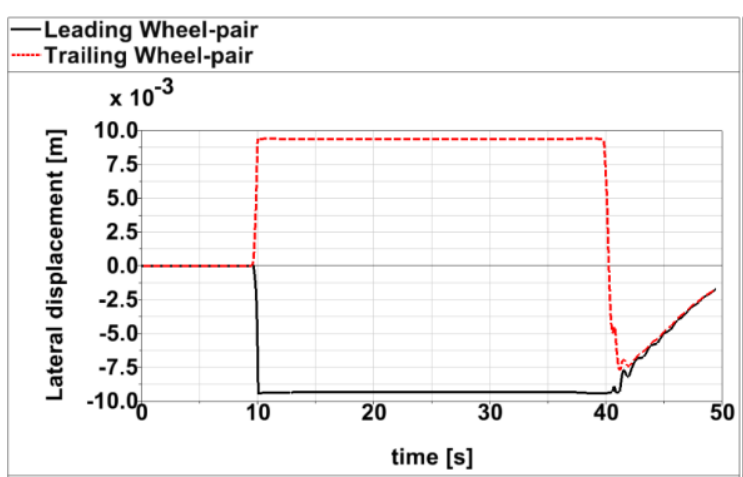

(a)

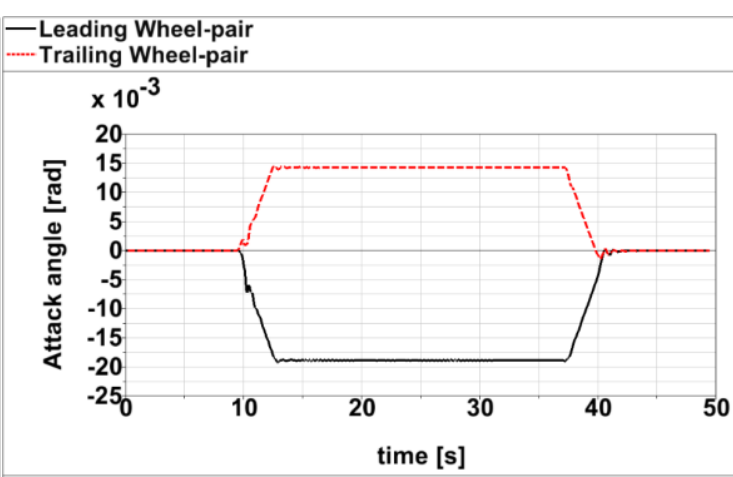

(b)

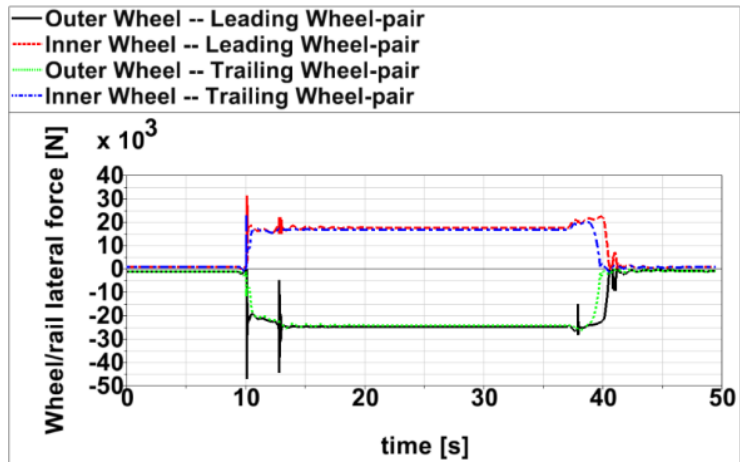

(c)

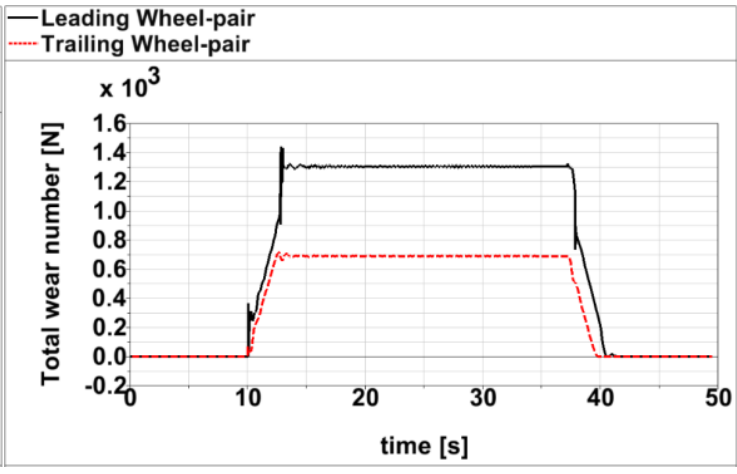

(d)

Figure 6. Dynamic responses of a passive two-axle IRW vehicle for a $250 \mathrm{~m}$ curve at 72 $\mathrm{km} / \mathrm{h}$ (without track irregularities). (a) Wheel-pair lateral displacement; (b) Attack angle; (c) Wheel/rail lateral force; (d) Wear index.

Considering the $250 \mathrm{~m}$ curve mentioned above, Figure 7 exhibits the same dynamic behaviour of the vehicle with the motor-steered dual-loop control scheme. Figure 7(a) shows the wheel-pair lateral displacements of the IRW vehicle. It can be seen that both wheel-pairs move outwards from the curve and the flange contacts are avoided. In Figure 7(b), the attack angles of the two wheel-pairs, which are very small and in the same direction, generate lateral creep forces to counterbalance the uncompensated centrifugal forces due to the cant deficiency. These phenomena represent the highly improved curving feature of the vehicle, which is virtually 'perfect-curving' [3] except the inevitable longitudinal creep forces due to motor steering. For curve negotiation, the IRW wheel-pair with the practical dual-loop control takes the advantage of the wheel tread conicity, i.e. the wheel radius difference, to negotiate the curve with almost the same rotational velocities for both wheels. From Figure $7(\mathrm{c})$, it can be noticed that the wheel/rail lateral forces are almost evenly distributed and considerably smaller than that of the passive system. In Figure 7(d), the total wear index for each axle is very small, since the flange contact can be avoided during the curve negotiation. Figure 7(e) and 
Figure 7(f) show the longitudinal and lateral creepages of the wheels with this motorsteered approach.

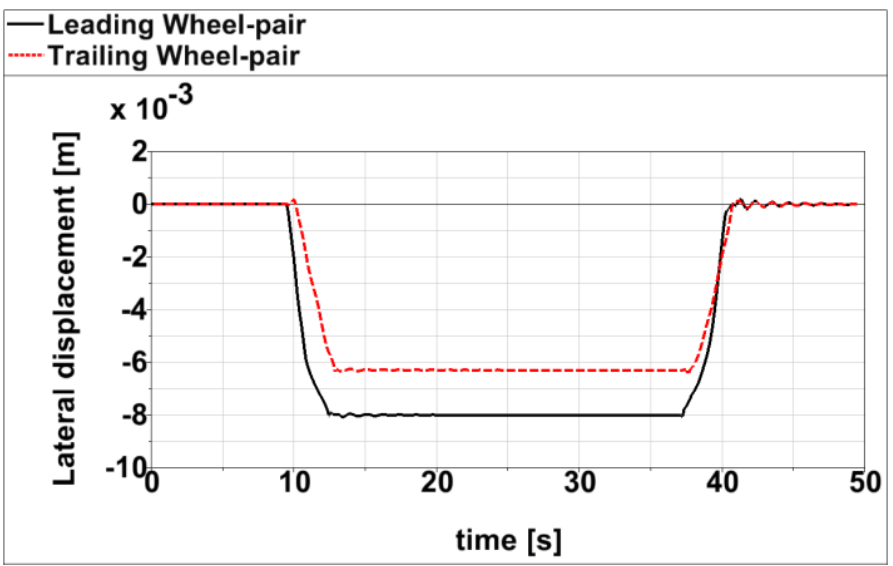

(a)

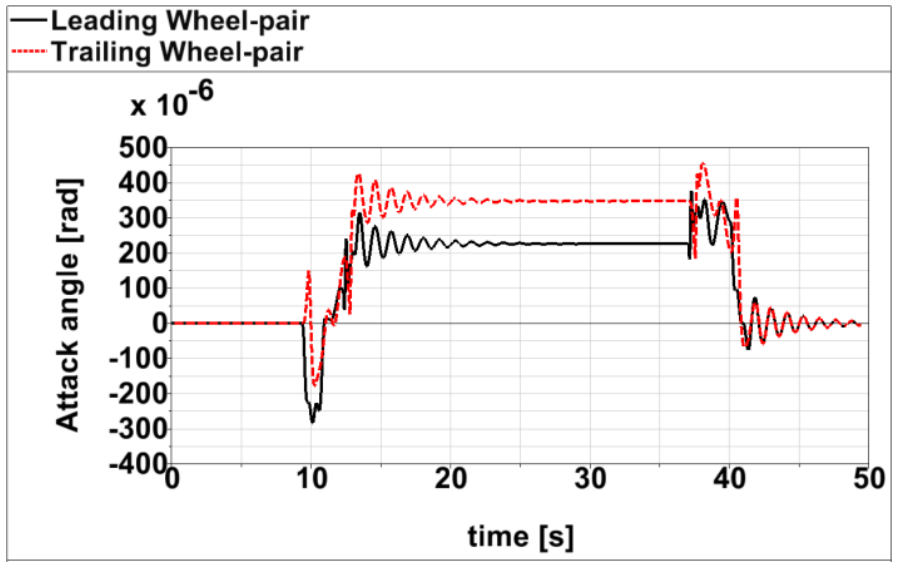

(b)

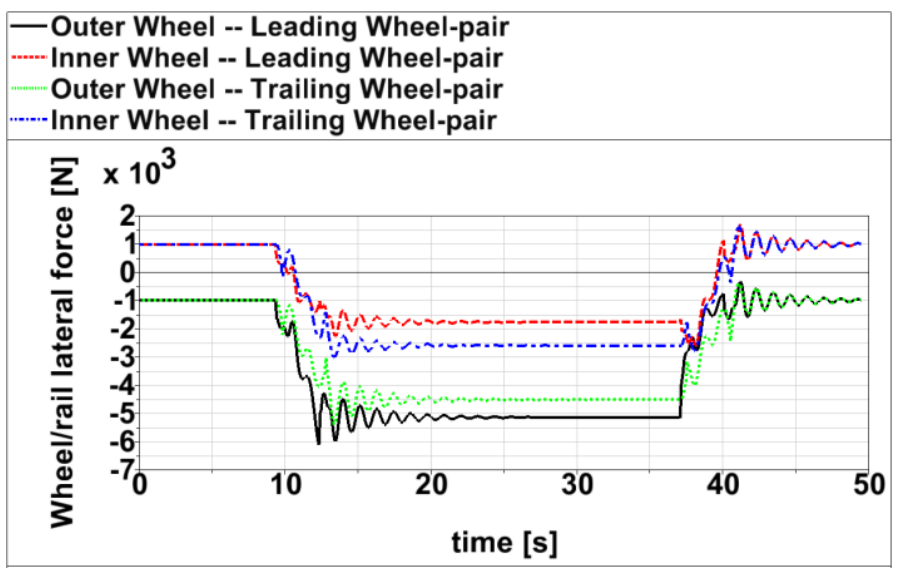

(c) 


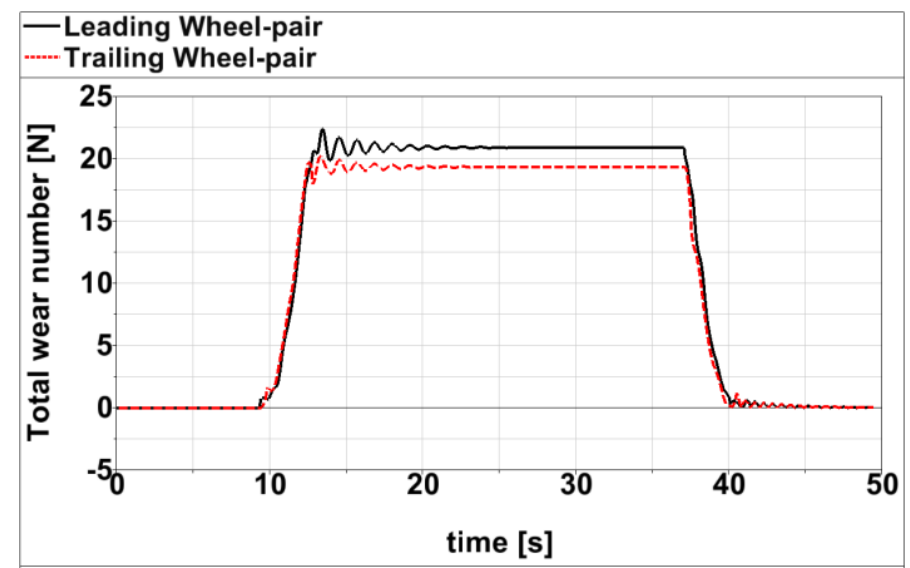

(d)

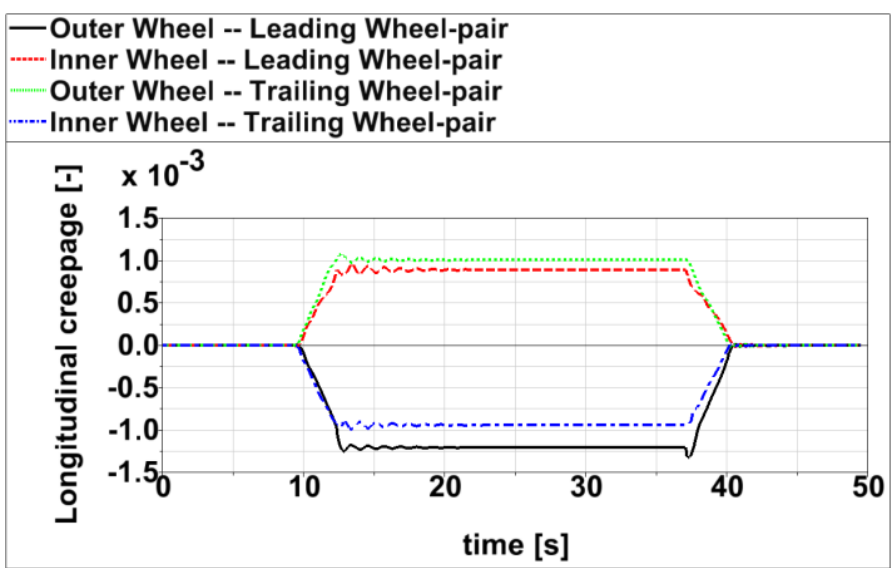

(e)

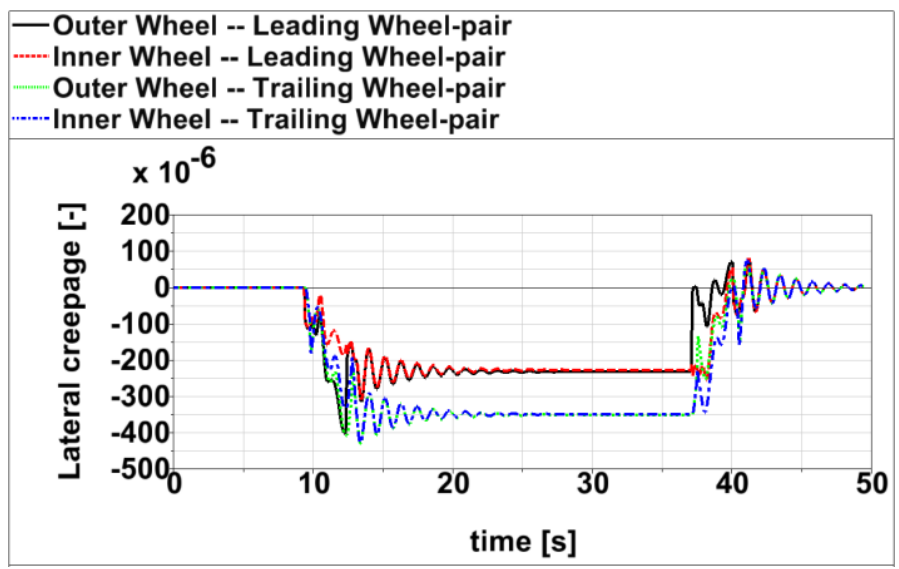

(f)

Figure 7. Dynamic responses of motor-steered two-axle IRW vehicle for a $250 \mathrm{~m}$ curve at $72 \mathrm{~km} / \mathrm{h}$ (without track irregularities). (a) Wheel-pair lateral displacement; (b) Attack angle; (c) Wheel/rail lateral force; (d) Wear index; (e) Longitudinal creepage; (f) Lateral creepage. 
Figure 8 shows the dynamic behaviour of the actuator-steered IRW vehicle with the dual-loop control scheme. From Figure 8(a) and Figure 8(b), the two wheel-pairs exhibit homogeneous behaviours with almost identical lateral displacements and attack angles. These behaviour represents the perfect curving feature of the vehicle, however, this control strategy realizes a strictly defined 'perfect-curving' performance because of the avoidance of the longitudinal creep in the curving. From Figure 8(c), it can be noticed that the wheel/rail lateral forces are similar to the ones of the motor-steered IRW vehicle, which are considerably smaller than that of the passive system. In Figure 8(d), the total wear index for each axle is even lower than that of the motorized IRW, since the curving wears caused by the longitudinal creep forces are eliminated. Figure 8(e) and Figure 8(f) show the longitudinal and lateral creepages of the wheels with this actuator-steered approach.

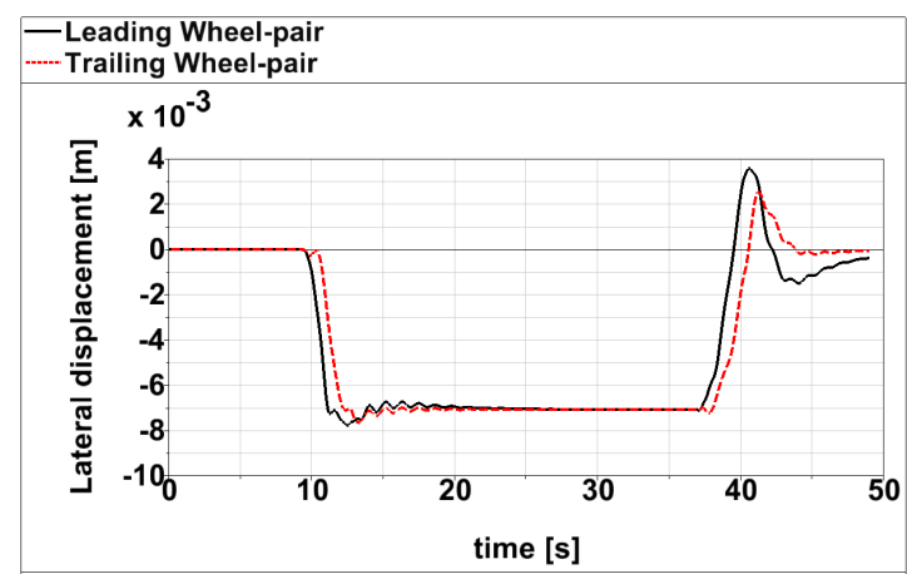

(a)

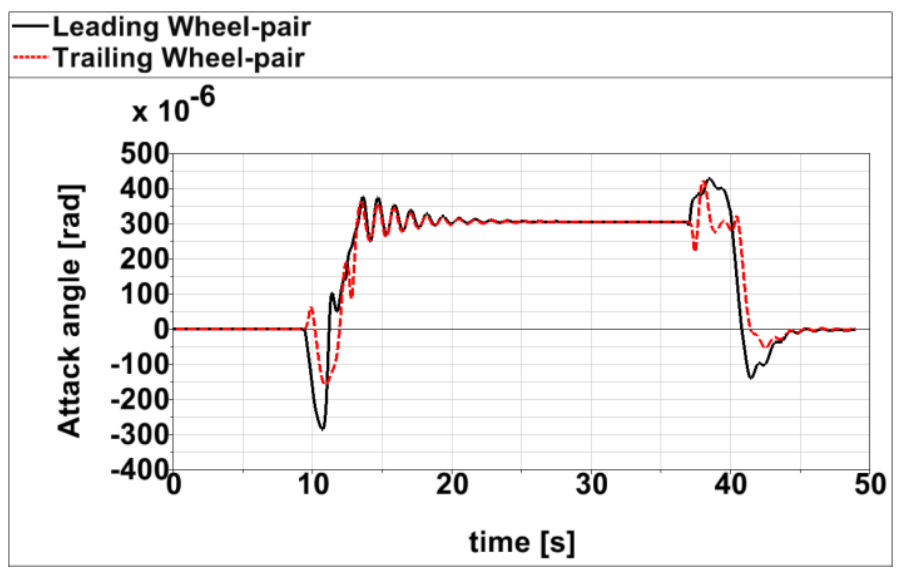

(b) 


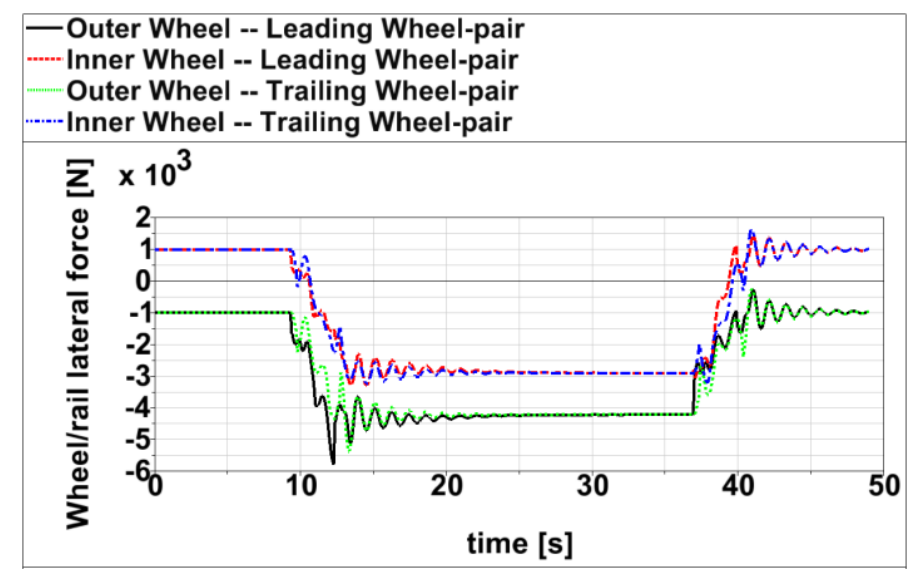

(c)

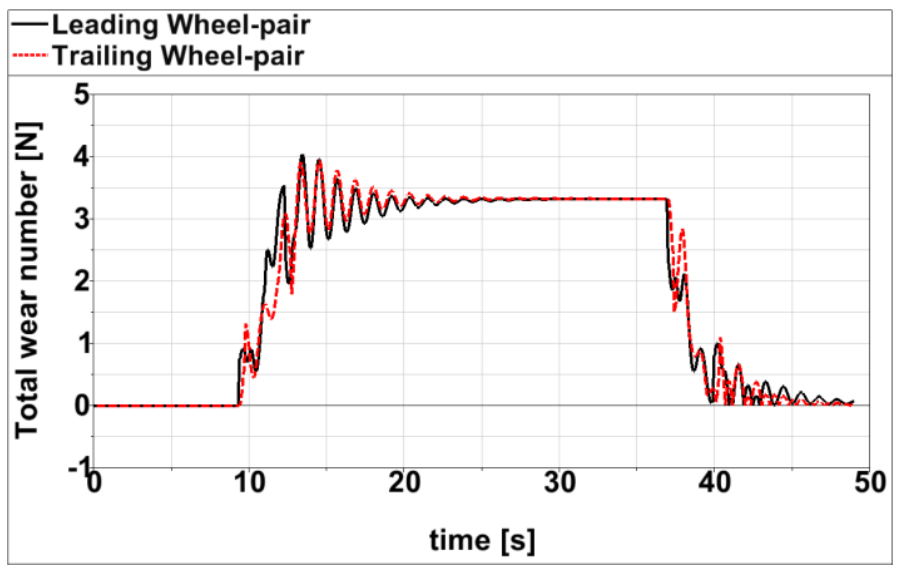

(d)

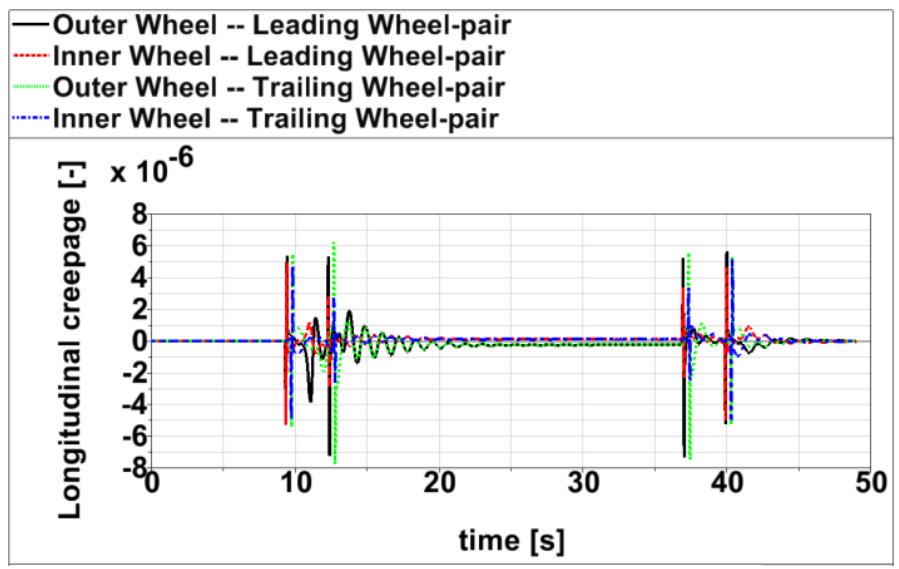

(e) 


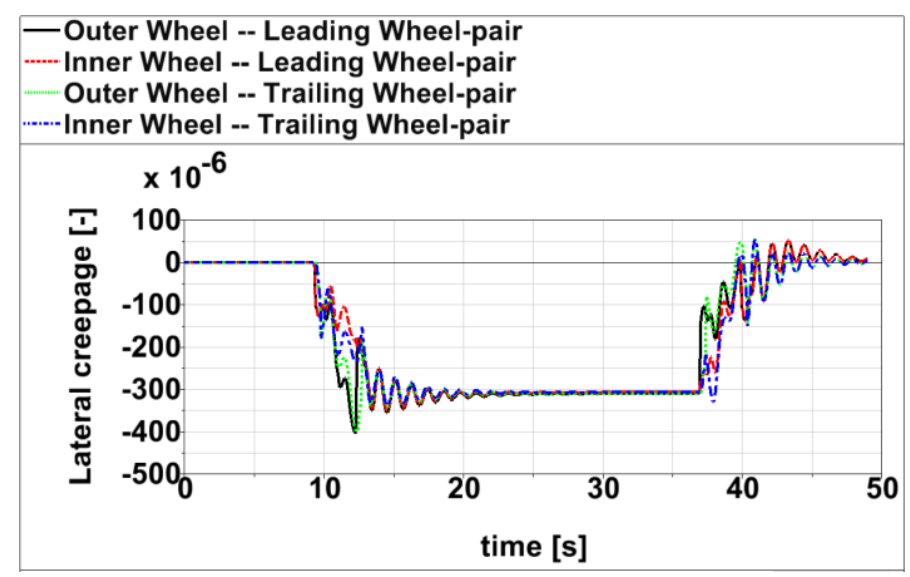

(f)

Figure 8. Dynamic responses of actuator-steered two-axle IRW vehicle for a $250 \mathrm{~m}$ curve at $72 \mathrm{~km} / \mathrm{h}$ (without track irregularities). (a) Wheel-pair lateral displacement; (b) Attack angle; (c) Wheel/rail lateral force; (d) Wear index; (e) Longitudinal creepage; (f) Lateral creepage.

For the same track case $(250 \mathrm{~m}$ curve at $72 \mathrm{~km} / \mathrm{h})$, Figure 9 demonstrates the active motor torques for the motor-steered IRW and the active yaw torques for the actuatorsteered IRW respectively. Here the track irregularity 'BR - Track110', which represents a poor track condition, is applied for both cases. It can be seen that, in both configurations, the opposite steering torques are applied to the leading and trailing wheel-pairs to realize their radial positions. From several simulations, it is also found that the required motor torque for the curve negotiation is proportional to the primary yaw stiffness. However, the yaw stiffness for the motor-steered solution cannot be reduced to a very low value. That is because with a very low yaw stiffness, the control system needs much higher motor torques to stabilize the wheel-pairs under the disturbance of the track irregularity and the resultant longitudinal creep torques can result in hunting motion. In contrast, the actuator-steered solution only just generates the longitudinal creep torques to negotiate the track irregularity. The actuation torque can be less sensitive to the track irregularity (see the spikes in Fig. 9). Since the wheel-pair can follow the track irregularity without leading to the hunting behaviour, the stability issue is mainly related to the control of the kinematic instability of the wheel-pair. As the ground-hook damping is adopted in this control method, a very low yaw stiffness is desirable both for the reduction of the steering torque in the curve and the running stability, since with a high yaw stiffness it is more difficult to realize the stability control with a ground-hook damping approach. In this 
sense, the curve negotiation and stability performances are no longer contradictory if this active control method is adopted.

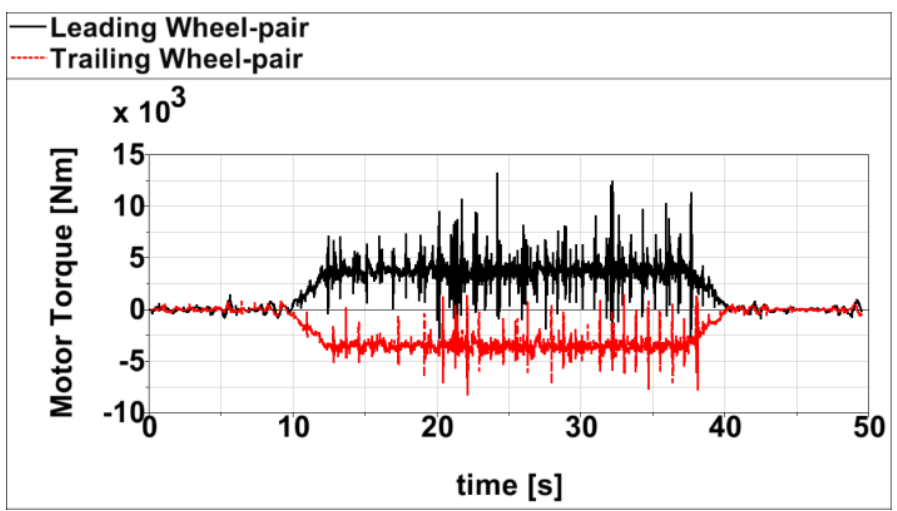

(a)

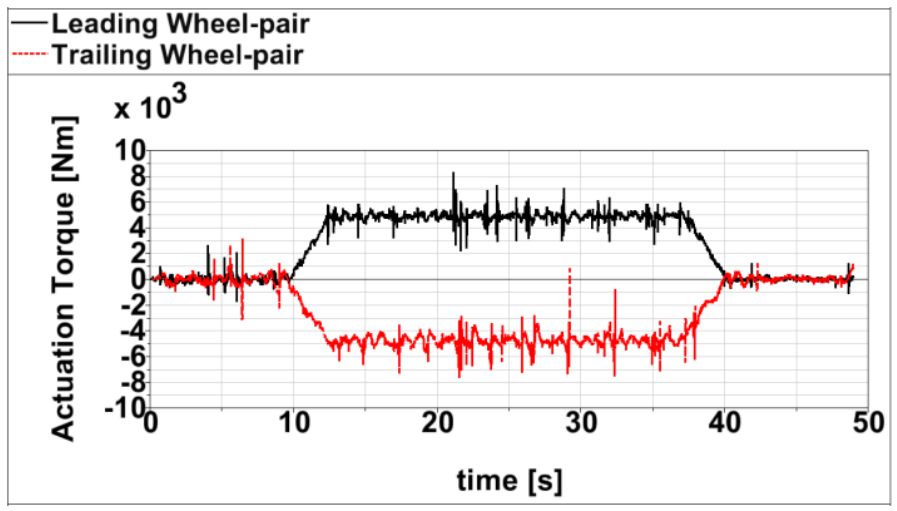

(b)

Figure 9. Steering torques for two-axle IRW vehicles for a $250 \mathrm{~m}$ curve at $72 \mathrm{~km} / \mathrm{h}$ with track irregularities. (a) Motor torque for motor-steered IRW; (b) Actuation torque for actuator-steered IRW.

Several curving simulations are carried out to investigate the curving performances of the motor-steered and actuator-steered control systems at different speeds, as shown in Table 2 and Table 3. Here the cant-deficiency speed refers to the curving speed with an uncompensated lateral acceleration of $0.65 \mathrm{~m} / \mathrm{s}^{2}$. From the curving indices shown in Table 2 and Table 3, the motor-steered control and actuator-steered control provide many homogenous results in the curve negotiations, such as the wheelpair attack angle, wheel/rail lateral force, etc. It is obvious that the wheel/rail interaction indices are very low with these active steering approaches compared with the values of the passive vehicle. The wear indices can be significantly reduced to very low levels with the active steering, especially for the actuator-steered control. It can also be noticed that the steering torque required to negotiate the curve is inversely proportional to the curve 
radius, however, the steering torque is almost independent of the vehicle speed. In Table 2 , the motor torques required for the steering functions are relatively small compared with the traction torque to achieve $1 \mathrm{~m} / \mathrm{s}^{2}$, approximately $2.5 \mathrm{kNm}$ in this case, which is only exceeded for the $250 \mathrm{~m}$ radius curve. In Table 3, the required yaw torque is very small because of the very low yaw stiffness and lack of slip between the two wheels of the wheel-pair.

Table 2. Curving indices for motor-steered IRW at cant-deficiency (d) and cant-balance (b) speeds.

\begin{tabular}{cccccccc}
\hline $\begin{array}{l}\text { Track case: } \\
\text { radius }[\mathrm{m}] / \\
\text { cant }[\mathrm{mm}]\end{array}$ & $\begin{array}{l}\text { Speed } \\
{[\mathrm{km} / \mathrm{h}]}\end{array}$ & $\begin{array}{l}\text { Wheel-pair } \\
\text { lateral } \\
\text { displacement } \\
{[\mathrm{mm}]}\end{array}$ & $\begin{array}{l}\text { Wheel/rail } \\
\text { lateral force } \\
{[\mathrm{kN}]}\end{array}$ & $\begin{array}{l}\text { Derailment } \\
\text { coefficient } \\
{[-]}\end{array}$ & $\begin{array}{l}\text { Attack } \\
\text { angle } \\
{[\mathrm{mrad}]}\end{array}$ & $\begin{array}{l}\text { Total } \\
\text { wear } \\
\text { number } \\
{[\mathrm{N}]}\end{array}$ & $\begin{array}{l}\text { Max. yaw } \\
\text { actuation } \\
\text { torque } \\
{[\mathrm{kNm}]}\end{array}$ \\
\hline \multirow{2}{*}{$250 / 150$} & $72.7(\mathrm{~d})$ & 8.0 & 5.1 & 0.08 & 0.35 & 20.9 & 3.8 \\
& $56.4(\mathrm{~b})$ & 7.8 & 1.66 & 0.032 & 0.16 & 19.1 & 3.7 \\
$400 / 100$ & $82.2(\mathrm{~d})$ & 6.8 & 4.5 & 0.07 & 0.38 & 10.2 & 2.45 \\
& $58.2(\mathrm{~b})$ & 6.6 & 1.01 & 0.019 & 0.09 & 7.56 & 2.36 \\
$600 / 80$ & $95.5(\mathrm{~d})$ & 5.8 & 4.7 & 0.08 & 0.41 & 6.4 & 1.69 \\
& $63.8(\mathrm{~b})$ & 5.4 & 0.92 & 0.017 & 0.07 & 3.7 & 1.6 \\
$1500 / 60$ & $142(\mathrm{~d})$ & 3.0 & 5.0 & 0.08 & 0.45 & 3.7 & 0.76 \\
& $87.3(\mathrm{~b})$ & 2.4 & 0.98 & 0.018 & 0.04 & 0.88 & 0.64 \\
\hline
\end{tabular}

Table 3. Curving indices for actuator-steered IRW at cant-deficiency (d) and cant-balance (b) speeds

\begin{tabular}{llllllll}
\hline $\begin{array}{l}\text { Track case: } \\
\text { radius }[\mathrm{m}] / \\
\text { cant }[\mathrm{mm}]\end{array}$ & $\begin{array}{l}\text { Speed } \\
{[\mathrm{km} / \mathrm{h}]}\end{array}$ & $\begin{array}{l}\text { Wheel-pair } \\
\text { lateral } \\
\text { displacement } \\
{[\mathrm{mm}]}\end{array}$ & $\begin{array}{l}\text { Wheel/rail } \\
\text { lateral force } \\
{[\mathrm{kN}]}\end{array}$ & $\begin{array}{l}\text { Derailment } \\
\text { coefficient } \\
{[-]}\end{array}$ & $\begin{array}{l}\text { Attack } \\
\text { angle } \\
{[\mathrm{mrad}]}\end{array}$ & $\begin{array}{l}\text { Total } \\
\text { wear } \\
\text { number } \\
{[\mathrm{N}]}\end{array}$ & $\begin{array}{l}\text { Max. yaw } \\
\text { actuation } \\
\text { torque } \\
{[\mathrm{kNm}]}\end{array}$ \\
\hline \multirow{2}{*}{$250 / 150$} & $72.7(\mathrm{~d})$ & 7.1 & 4.21 & 0.067 & 0.31 & 3.33 & 4.9 \\
& $56.4(\mathrm{~b})$ & 7.1 & 0.94 & 0.017 & 0.087 & 0.82 & 4.8 \\
$400 / 100$ & $82.2(\mathrm{~d})$ & 5.9 & 4.14 & 0.066 & 0.33 & 2.89 & 3.2 \\
& $58.2(\mathrm{~b})$ & 5.9 & 0.67 & 0.013 & 0.069 & 0.57 & 3.0 \\
$600 / 80$ & $95.5(\mathrm{~d})$ & 4.4 & 4.53 & 0.072 & 0.38 & 2.83 & 2.2 \\
& $63.8(\mathrm{~b})$ & 4.3 & 0.77 & 0.014 & 0.062 & 0.46 & 2.0 \\
$1500 / 60$ & $142(\mathrm{~d})$ & 1.8 & 4.84 & 0.078 & 0.43 & 2.94 & 0.98 \\
& $87.3(\mathrm{~b})$ & 1.8 & 0.98 & 0.018 & 0.032 & 0.24 & 0.81 \\
\hline
\end{tabular}




\subsection{Stability performance}

To test the vehicle stabilities with the active control approaches, the high-speed operational conditions are utilized considering the straight track. Here the high-speed track irregularity 'BR - Track 270' with a low disturbance is adopted in the following simulations. For high-speed operation, larger controller gains are utilized in the control loops for the fast responses. Figure 10 exhibits the wheel-pair displacements of the motorsteered IRW vehicle at the speed of $300 \mathrm{~km} / \mathrm{h}$. In Figure 10, although the wheel-pairs oscillate laterally with large amplitudes, the hunting motion does not appear at this speed. However, the required motor torques for the stability control are very high compared with the values for conventional motors. Since the stability control is realized by the differential motor torques, the large motor torques are needed to maintain the axle-bridge in a balanced position at high frequencies. Due to its stability issue and technical limitations, the motor-steered approach is preferable for the lower speed applications.

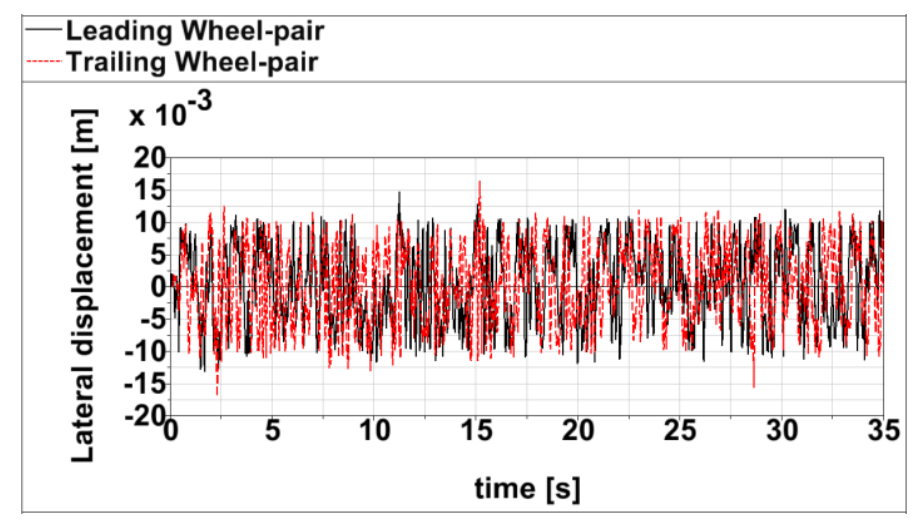

Figure 10. Wheel-pair lateral displacements of motor-steered IRW vehicle at $300 \mathrm{~km} / \mathrm{h}$.

Figure 11 demonstrates the wheel-pair displacements and the actuation torques for the actuator-steered IRW vehicle at $300 \mathrm{~km} / \mathrm{h}$ with the same track case. In Figure 11(a), the vehicle exhibits a stable running behaviour at this speed. The wheel-pair lateral displacement is much smaller than that of the motor-steered IRW. The stability by the actuator-steered approach is relatively easy to guarantee because of a lack of the hunting motion. From Figure 11(b)-(c), it is found that the active yaw torques, with an RMS value of $1.3 \mathrm{kNm}$, are within the capacities of the current actuator techniques. Additionally, it is also found that by utilizing lightweight IRW wheel-pairs, it is possible to improve the stability performances of the vehicle at high speeds with even lower actuation torques. 


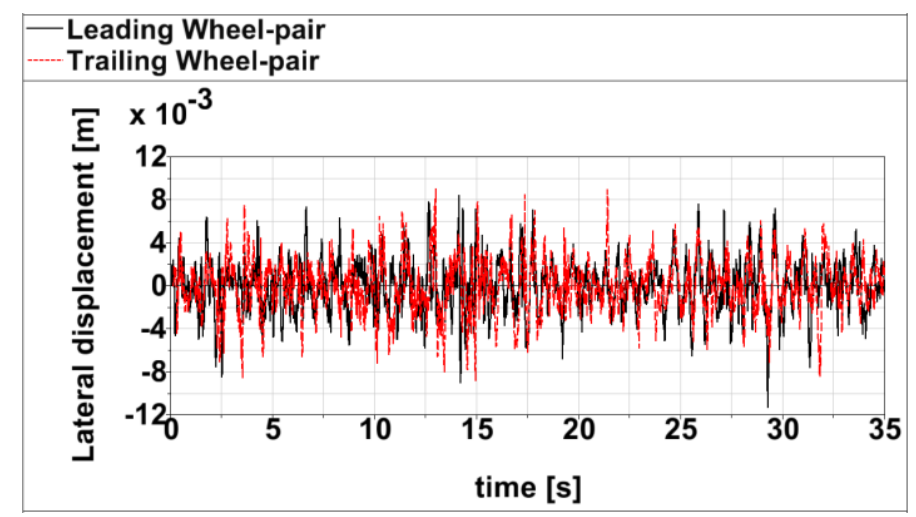

(a)

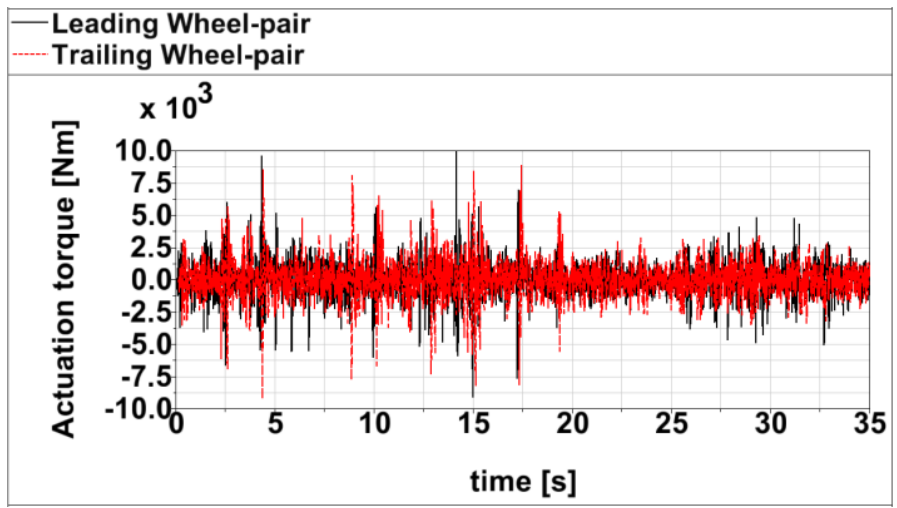

(b)

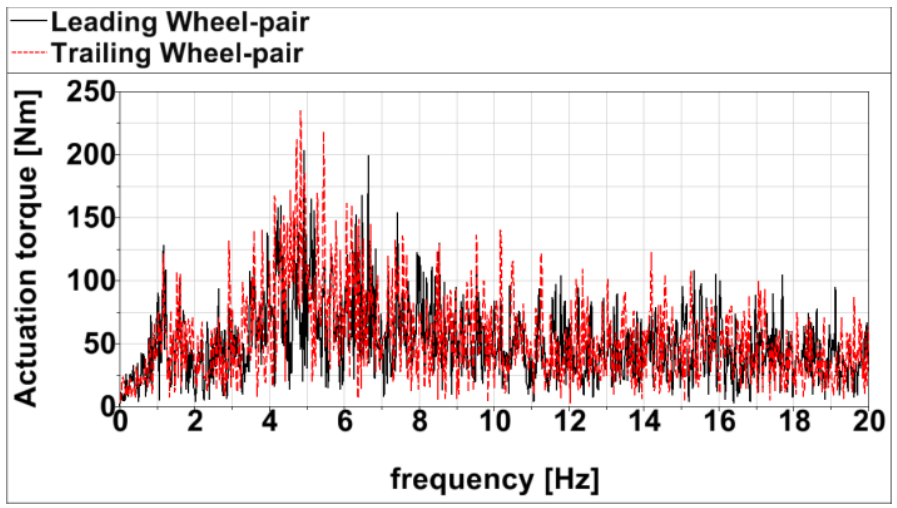

(c)

Figure 11. Wheel-pair lateral displacements and actuation torques of actuator-steered IRW vehicle with conventional wheel-pairs at $300 \mathrm{~km} / \mathrm{h}$. (a) Wheel-pair lateral displacements; (b) Actuation torques; (c) Spectra of actuation torques. 


\section{Conclusions and Outlooks}

This paper presents active control strategies for motor-steered and actuator-steered IRW systems. In these control approaches, only the wheel rotational velocities and the yaw angular velocity of the axle-bridge need to be measured for the control system, which can be realized by tachometers and gyroscopes respectively. With these vehicle-based observations, the more difficult measurements for the quantities between the wheel/rail interfaces and the feedforward input of the track database can be avoided. Dual-loop control methods are utilized for the active systems, including a differential control for the steering function and a ground-hook yaw-rate control for the stability purpose.

The dual-loop control strategies for the motor-steered and actuator-steered IRWs are validated by the numerical simulation results. From the simulation results, both control methods exhibit perfect-curving performances with very low wear and wheel/rail forces. This is due to the differential control in commanding the two wheels to rotate at almost the same rotational speed at low frequencies with a suitable wheel radius difference for the curve. The instability caused by the differential control can be solved by the yaw-rate control loop, which is based on a ground-hook damping approach. The active torques/forces needed for the motor-steered and actuator-steered systems are reasonable and within typical capacities of the current motors and actuators. Since the longitudinal creep torque is involved in the steering and balancing functions for the motor-steered approach, the stability of the vehicle could be an issue, which may require a suitable yaw stiffness. Because the motor-steered approach does not need additional actuators for the motorized IRW vehicle, this steering scheme can be readily applied to a motorized IRW vehicle at a low cost. For the actuator-steered method, only the lateral creep force contributes to the steering of wheel-pair. The longitudinal creep torque and the resultant hunting motion can be eliminated. Associated with the ground-hook damping, it is preferable to adopt a very low yaw stiffness to accomplish good curve negotiation and stability simultaneously with less actuation powers, leading to an excellent overall vehicle dynamic performance.

This research is fundamentally oriented towards the control strategies of IRW systems. The further developments of the proposed control concepts could focus on the application issues of control systems. These questions include the signal processing of the measured signals and the investigations of realistic actuator behaviours. For instance, the time delay due to the possible low-pass filtering of the measured signal needs to be 
taken into consideration in the real control system, where a phrase advance controller could be adopted. In addition, the realistic actuator behaviours, such as the power rating, torque/force restriction and time delay, are also the key issues of the designed control systems, where more detailed specifications and modelling of wheel motors and hydraulic actuators are needed. These questions could be discussed on the next stage of this research.

\section{Acknowledgement}

The authors acknowledge the EU 'Shift2Rail' RUN2Rail project for the financial support to this research under grant agreement No. 777564.

\section{References}

[1] Goodall R, Li H. Solid axle and independently-rotating railway wheelsets - a control engineering assessment of stability. Vehicle Syst Dyn. 2000; 33(1): 5767.

[2] Goodall RM, Mei TX. Active suspensions. In: Iwnicki S, editor. Handbook of railway vehicle dynamics. Boca Raton: CRC Press; 2006. p. 327-357.

[3] Goodall RM, Bruni S, Mei TX. (2006) Concepts and prospects for actively controlled railway running gear. Vehicle Syst Dyn. 2006; 44(sup1): 60-70.

[4] Bruni S, Goodall R, Mei TX, et al. Control and monitoring for railway vehicle dynamics. Vehicle Syst Dyn. 2007; 45(7-8): 743-779.

[5] Mei TX, Goodall RM. Recent development in active steering of railway vehicles. Vehicle Syst Dyn. 2003; 39(6): 415-436.

[6] Mei X, Li H, Goodall RM, et al. Dynamics and control assessment of rail vehicles using permanent magnet wheel motors. Vehicle Syst Dyn. 2002; 37(sup1): 326337.

[7] Kurzeck B, Heckmann A, Wesseler C, et al. Mechatronic track guidance on disturbed track: the trade-off between actuator performance and wheel wear. Vehicle Syst Dyn. 2014; 52(sup1): 109-124.

[8] Mei TX, Goodall RM. Wheelset control strategies for a two-axle railway vehicle. Vehicle Syst Dyn. 1999; 33(sup1): 653-664.

[9] Mei TX, Goodall RM. Practical strategies for controlling railway wheelsets independently rotating wheels. J Dyn Sys Meas Control. 2003; 125(3): 354-360. 
[10] Perez J, Mauer L, Busturia JM. Design of active steering systems for bogie-based railway vehicles with independently rotating wheels. Vehicle Syst Dyn. 2002; 37(sup1): 209-220. 